\title{
The halo sign during a percutaneous nephrolithotomy puncture
}

\author{
M.G. Pradeepa, DNB; Maneesh M. Sinha, MCh; Krishnaprasad Tyagi, DNB
}

NU Hospitals, Karnataka, India

Cite as: Can Urol Assoc J 2016;10(3-4):E130. http://dx.doi.org/10.5489/cuai.3353

W hen attempting to gain pelvicalyceal system (PCS) access for a percutaneous nephrolithotomy (PCNL), the initial puncture is the most important step. Free efflux of urine through the needle or on aspiration is an absolute sign of the needle tip being in the PCS. The trouble occurs when the needle tip appears to be in the system, but no urine can be aspirated. Attempts to pass a guidewire are unsuccessful. These are moments of anxiety all urologists have experienced. This is where the halo sign is a useful guide to the location of the needle tip.

The halo sign is elicited only during contrast fluoroscopyguided needle punctures of the PCS (Fig. 1). Under these circumstances, when the needle lies outside, immediately external and parallel to the PCS, abutting on its wall, movement of the needle tip gives rise to a pale sphere of contrast around the needle tip (Fig. 2), akin to a halo. The needle tip may be anterior or posterior to the PCS, but is immediately adjacent to it.

Under these conditions, the tip of the needle on movement displaces contrast from its immediate surroundings; the region of interest, in this case, being the PCS. This displacement results in a radiolucent area around the needle tip. The practical use of this identification is that the surgeon must not attempt to place a guidewire, or continue with aspirations or random movements of the needle, as it can injure the vasculature. Minimal manipulation in the right direction will achieve success in puncturing the PCS.

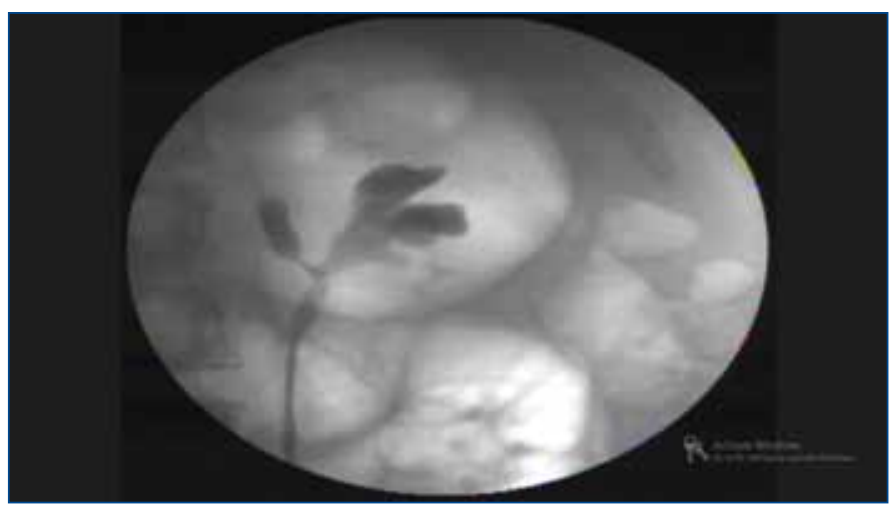

Fig. 1. Dilated calyx.
For obvious reasons, this halo is not visible in grossly dilated systems. It is seen only when the needle is able to displace a fluoroscopically discernible amount of contrast from the adjacent PCS.

Clinical radiology abounds with a number of situations where the halo sign has been described. Examples are seen on computed tomography of the chest in aspergillus infection and on ultrasound imaging for temporal arteritis or in fetal death..$^{1-3}$ While most of these have diagnostic relevance, the halo around the PCNL needle is of practical importance, as it can save both time and unnecessary effort during PCNL punctures.

Competing interests: The authors declare no competing financial or personal interests.

This paper has been peer-reviewed.

\section{References}

1. Schmidt W, Kraft H, Vorpahl K, et al. Colour duplex ultrasonography in the diagnosis of temporal arteritis. N Engl J Med 1997;337:1336-42. http://dx.doi.org/10.1056/NEJM199711063371902

2. Shaff MI. An evaluation of the radiological signs of fetal death. S Afr Med J 1975;49:736-8.

3. Lee YR, Choi YW, Lee KJ, et al. CT halo sign: The spectrum of pulmonary diseases. Br J Radiol 2005;78:862-5. http://dx.doi.org/10.1259/bir/77712845

Correspondence: Dr. M.G. Pradeepa, NU Hospitals, Karnataka, India; dr.pradeep@nutrust.net

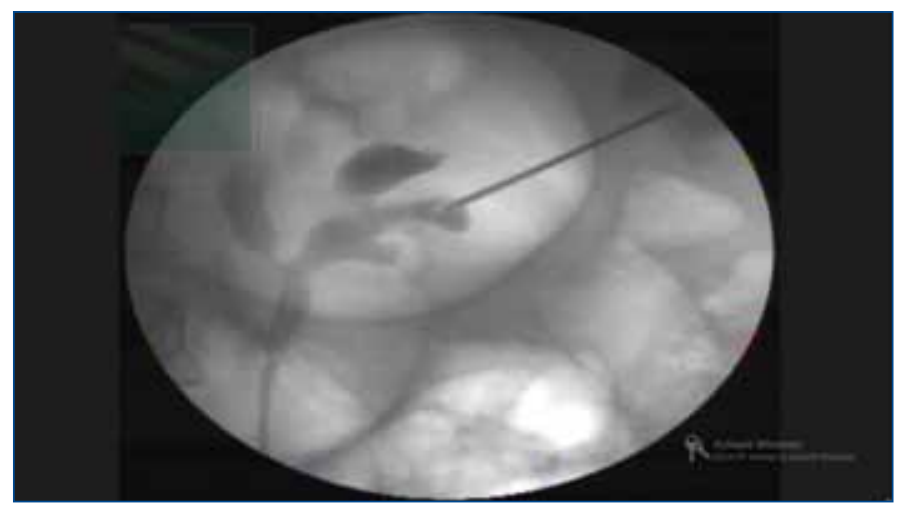

Fig. 2. Halo sign. 